\title{
Shakespeare et les Contes sans fin du mal
}

\section{Margaret Jones-Davies}

\section{(2) OpenEdition \\ Journals}

\section{Édition électronique}

URL : http://journals.openedition.org/shakespeare/1145

DOI : 10.4000/shakespeare.1145

ISSN : 2271-6424

Éditeur

Société Française Shakespeare

Édition imprimée

Date de publication : 1 novembre 1997

Pagination : 77-100

Référence électronique

Margaret Jones-Davies, "Shakespeare et les Contes sans fin du mal », Actes des congrès de la Société française Shakespeare [En ligne], 15 | 1997, mis en ligne le 01 janvier 2007, consulté le 06 mai 2019. URL : http://journals.openedition.org/shakespeare/1145; DOI : 10.4000/shakespeare.1145 


\section{S H A K E S P E A R E E T L ES CONTES S A N S}

\section{F I N D U M A L}

Quand s'abat sur elle la colère de Dieu, la vie de l'homme est comparable à un conte, nous dit le Psaume 90 :

For all our days are passed in thy wrath

We spend our years as a tale that is told.

Shakespeare emprunte à la Bible cette idée, la répète, l'interprète, l'amplifie. Qu'est-ce qu'un conte, sinon l'art de trouver une fin au récit de la vie ? Si la vie devient un conte que l'on a raconté - notons la forme passive «that is told» - si elle se ferme comme un livre, alors les années se déroulent sans fin sans que soient jamais plus ressentis les bienfaits de la Providence, symbole d'ouverture sur l'espoir de la grâce, et l'homme s'enferme dans l'espace clos du Destin. C'est la dure leçon de Macbeth, qui définit la vie comme un conte et se voit «cabin'd, cribb'd, confined...» (Macbeth, III.4.23).

Ce paradoxe d'une vie circonscrite dans les limites d'un conte qui voit le temps s'étirer de lendemain en lendemain à l'infini, décrit la tragédie de l'homme.

Tomorrow and tomorrow and tomorrow

Creeps in this petty pace from day to day

To the last syllable of recorded time.

(Macbeth, V.5.19-21) 
De manière répétée et donc signifiante, la tragédie shakespearienne se termine sur un conte, non pas comme on pourrait le croire, pour boucler l'intrigue, mais au contraire et paradoxalement pour en dire l'inachèvement. Car les contes à la fin des tragédies de Shakespeare restent en suspens, comme on imagine que reste en suspens le «poem unlimited» avec lequel Polonius clôt la longue liste des genres littéraires dans Hamlet (II.1.400). La fin d'Othello est le conte interrompu, donc jamais fini du «malignant and turban'd Turk» (Othello, V.2.356-357). Les derniers mots de King Lear racontent de curieuses histoires de papillons dorés qui symbolisent comme dans Coriolanus la quête infinie d'un pouvoir jamais atteint ${ }^{1}$, et Richard II imagine Isabelle racontant chaque soir «sad stories of the death of kings» (III.2.156), le crime de Brutus se répétera à l'infini sur toutes les scènes du monde (Julius Caesar, III.1.111-113) et Macbeth voit sa vie se dissoudre dans le conte infiniment répété des jours, qui ne cessent de se succéder (Macbeth, V.5.18-28).

\section{Des tragédies inachevées}

Pour Shakespeare, les contes sont sans fin, car ils expriment de manière privilégiée cette crise, dont Frank Kermode propose la célèbre théorie dans The Sense of an Ending, 1966. Paul Ricœur la résume en une phrase dans le 2ème volume de Temps et Récit : «La crise ne marque pas l'absence de toute fin mais la conversion de la fin imminente en fin immanente ${ }^{2} \gg$. La fin n'est plus à prévoir, la fin ne cesse de finir, elle nous enferme dans une mortalité sans issue.

L'Apocalypse avait promis la fin des temps, comme une récompense de complétude, un achèvement qui donnerait un sens à ce récit qu'est notre vie. Mais quelle déception à la fin du parcours ; c'est une fin qui ne se termine pas : «is this the promised end?», se demande Kent dans King Lear (V.3.263). Cette fin qu'il découvre n'en est pas une. Elle n'a rien de l'apaisement du «telos», de cette découverte de ce que Northrop Frye appelait dans sa description de la dernière phase du symbole, «the still center of the order of words» ${ }^{3}$. C'est une fin éternellement reportée. La crise est donc cette sempiternelle transition que Kermode fait remonter à l'interprétation des trois ans et demi du règne de la Bête, qui précèdent, dans l'Apocalypse, les derniers jours. Joachim de Flore (1202), l'un des rares millénaristes qui sut se faire accepter par l'Autorité, élabora une théorie de l'histoire et de cette transition, ce passage vers ce qu'il appelait le «troisième royaume» ou «IIIe Reich», aux connotations devenues sinistres depuis que, toujours selon Kermode, le National-Socialisme intégra des aspects de la théorie de Joachim de Flore dans son idéologie ${ }^{4}$

La tragédie n'est donc pas une comédie qui finirait mal, mais l'espace théâtral pour un temps qui ne finirait pas, la forêt sans horloge de As You Like It, ou ces océans du temps, ces «tides of time» au rythme sans cesse 
renouvelé - et inversement, la comédie, puisqu'elle est censée terminer le déroulement incessant du mal, ne pourrait présenter comme seule conclusion que celle de Benedick dans Much Ado About Nothing:

Man is a giddy thing and this is my conclusion.

$(\mathrm{V} .4 .107)$

conclusion qui dit mieux que toute autre la crise, son incomplétude - jusque dans la comédie même.

Shakespeare nous frustre donc de notre désir de fin, et de la manière la plus paradoxale qui soit. Au rêve achevé du millenium, s'oppose donc le récit toujours reporté des mille et une nuits.

Je tenterai d'abord de montrer que la caractéristique majeure attribuée par Shakespeare au mal, est l'éternité, que cela explique qu'il choisisse comme support privilégié pour le dire, le conte, et que cela révèle un parti pris philosophique, voire même politique.

\section{Le mal sans fin}

L'adjectif «eternal», nous dit le O.E.D. $\left(\mathrm{N}^{\circ} 7\right)$, peut signifier «infernal». Deux citations shakespeariennes viennent illustrer cette définition: «Th'eternal devil» (Julius Cassar, I.2.160) ; «Some eternal villain»(Othello, IV.2.131). On peut citer aussi Hamlet

$$
\text { O proud Death }
$$

What feast is toward in thine eternal cell, $[\ldots]^{5}$

$$
\text { (V.2.317-318) }
$$

Malcolm se sert de l'adjectif «confineless» pour qualifier les maux qu'il porte en lui «confineless harms» (IV.3.54). Lorsque le fantôme apparaît dans Hamlet, c'est comme conteur du mal éternel de l'enfer :

I could a tale unfold whose lightest word Would harrow up thy soul, freeze thy young blood

Make thy two eyes like stars start from their spheres

Thy knotty and combined locks to part

And each particular hair to stand on en

Like quills upon the fretful porcupine

But this ETERNAL BLAZON must not be

To ears of flesh and blood. 
héraldique se limite au sens de proclamation).

L'éternité du mal n'allait pas de soi. Dans ses récentes Histoires du Mal, Bernard Sichère analyse la position complexe des Pères de l'Église sur cette question controversée ${ }^{6}$. L'idée de l'éternité de la damnation finira par être imposée vers 250 après J.C., et même Origène qui croyait dans la doctrine de l'apocatastase (le monde retrouvera sa pureté originelle) finira par admettre dans Contre Celse, une conception éternelle de l'Enfer pour que le peuple garde vivante la conscience du mal ${ }^{7}$. L'éternité de la damnation est donc d'abord un concept à usage politique, puisque les élites pouvaient se permettre d'en douter. Il était en effet vital pour l'Église de briser les rêves millénaristes qui s'appuyaient sur le chapitre 20 de l'Apocalypse, où saint Jean imagine les mille ans libérés du règne de Satan - véritable aubaine pour les utopistes politiques. Dans La Fin des Temps, Georges Duby ${ }^{8}$ a très bien montré l'aspect contestataire du millénarisme et l'intérêt qu'avait l'Église de le contrer, d'où le revirement d'Origène et les écrits de son disciple Denys d'Alexandrie et de saint Augustin (XXe livre de La Cité de Dieu). "L'Église taxe d'orgueil, de péché contre l'esprit les téméraires qui prétendaient sonder le mystère des intentions divines, prévoir le jour et l'heure» ${ }^{9}$.

A l'époque de Shakespeare, l'imaginaire apocalyptique utilisé pour suggérer une époque purifiée du mal est sévèrement contrôlé. Il ne faut pas que l'on puisse prévoir une fin au mal sur terre. On pense aux lois de 1541 contre les prophéties, et aux exécutions capitales à l'encontre de ceux qui cherchaient à transcrire en termes politiques les prophéties de Merlin, toutes empreintes d'images apocalyptiques de dragons et d'aigles ${ }^{10}$. On pense à la condamnation de la prophétie de la taupe dans 1 Henry IV (III.1.149) ou à la relativisation dans The Tempest de la vision apocalyptique d'un nouveau monde (V.1.183). Que le mal puisse s'arrêter, que le règne de Satan sur terre soit aboli est une idée à écarter. On ne manque pas de rappeler la phrase de l'Épître aux Thessaloniciens : «Lorsqu'ils se diront paix et sécurité, la mort s'abattra sur eux» ${ }^{11}$ (I.5.3), que l'on peut considérer comme une source du célèbre vers «security is mortals' chiefest enemy» dans Macbeth (III.5.32-33). L'absence de mal est toujours suspecte : c'est Iago qui dit «I lack iniquity / Sometimes to do me service» (Othello, I.2.3-4). Et Y. Peyré rappelait que l'utopie de Gonzalo (The Tempest, II.1.145-154) est une rêverie qui ignore l'existence du $\mathrm{mal}^{12}$.

L'œuvre de Shakespeare garde de nombreuses traces de cette conception d'un mal infini, anti-millénariste que les autorités cherchaient à imposer. Et le corollaire de cette prévention contre l'orgueil de l'homme est l'adhésion aux thèses du déclin du monde (contre la doctrine aristotélicienne de son éternité), que l'on trouve sans cesse en cette fin du XVIe siècle et en particulier chez Shakespeare (Julius Caesar, Macbeth et King Lear). La terre, nous devons nous en souvenir, est tout entière vouée au déclin - l'homme ne doit pas 
espérer un bonheur terrestre utopique - et les fantômes qui la visitent ont des contes sans fin à raconter sur la damnation éternelle ${ }^{13}$. C'est ainsi que les autorités défendaient la morale.

\section{La généalogie du mal dans la morale shakespearienne et son} expression dans les contes

Le discours des autorités révélait deux priorités que l'on retrouve sans cesse dans la généalogie de la morale shakespearienne : à savoir que le mal sur terre, comme la damnation était éternel et que le jugement de l'homme avait le pouvoir de contrôler sa volonté. Il s'agissait donc de garder vivante la conscience morale en écrasant dans l'homme les deux sources de son orgueil. Depuis le moyen âge, un grand débat opposait sur ce sujet les thomistes aux scotistes. Saint Thomas avait prudemment mis la volonté sous la dépendance du jugement : on ne pouvait donc jamais de plein gré vouloir le mal. Si l'on croit vouloir le mal c'est suite à une défaillance du jugement, fragilisé par les assauts de l'ignorance ou des passions. La tradition nominaliste au contraire avec Duns Scot, puis Ockham, donnait à la volonté une autonomie, un pouvoir infini qui pouvait balayer les raisons morales et une claire conscience de ce qui est le bien ${ }^{14}$

Les échos de ce débat se font entendre dans le livre 4 du Courtisan de Castiglione (1528,tr. 1561). Signor Ottaviano le dit: «Men never choose evil deliberately but are deceived by a certain semblance of good» ${ }^{15}$. Et le Signor Gaspard répond : «Yet there are many who fully understand that they are doing evil, and still do it ; and this is because, like thieves and murderers, they are more conscious of the pleasures of the moment than of the punishment they fear in the future».

L'une des pièces de Shakespeare s'inspire de ce texte et des termes de ce débat au point qu'on peut la considérer comme un véritable traité sur le mal, c'est Much Ado About Nothing. Une source majeure de Much Ado About Nothing est un conte, une version de Barbe Bleue à laquelle Shakespeare se réfère comme «the old tale»(I.1.200). Au centre de ce conte est le Mal, insondable, répétitif, infini. En racontant le Mal, le conte l'explique, en arrête le déroulement, réussit à y mettre un terme. C'est en effet dans un conte, le récit d'un rêve qu'elle prétend avoir fait que Lady Mary dénonce les agissements pervers de Mr Fox, qui découpe en morceaux les femmes qu'il reçoit chez lui. Ayant visité la maison du criminel, à l'insu de celui-ci, et découvert les squelettes qu'elle recèle, témoin aussi du dernier crime commis, Lady Mary raconte ces faits comme s'il s'agissait de faits fictifs, rêvés au cours d'un banquet où se trouve Mr Fox. La conteuse ponctue son réci d'interjections rassurantes pour marquer l'irréalité des horreurs qu'elle raconte : «It is not so, nor it was not so...», jusqu'au dernier épisode, lorsque, brandissant la main coupée de la dernière victime, elle change en affirmation 
les dénégations précédentes : «But it is so, and it was so, and here the hand I have to show».

Le conte a le pouvoir ici de mettre un terme au mal. C'est le pouvoir des contes des mille et une nuits. Loin d'être l'expression du mal, le conte est, comme le dit Harold Weinrich, dans son analyse des contes médiévaux «la forme que prend la sagesse pour mûrir en décision» ${ }^{16}$. Il imprime au désordre du mal une logique, la logique tripartite - les répétitions ternaires émaillent le conte (voir appendice) - propre aussi bien à la grammaire qu'à l'Ordo Rerum comme le remarquait Quintilien : «En effet, de même qu'il y a trois temps, de même l'ordre du monde est constitué de trois moments : tout a un commencement, un déroulement, une fin ; ainsi : au début est la querelle, puis le combat, puis le meurtre ${ }^{17}$ »"

Or, ce n'est pas la capacité d'ordonner le monde que Shakespeare retient du conte de Lady Mary, mais simplement les fréquentes références aux refrains «It is not so» / «It is so» pour souligner la dialectique entre vérité et mensonge que $M u c h$ Ado orchestre de manière si magistrale.

En effet, la comédie met en scène des personnages qui font un usage différent du conte. Dans cette pièce, ils sont les narrateurs de contes comme les personnages du Courtisan dont ils s'inspirent tant. Ils nous permettent de comprendre l'articulation entre une réflexion philosophique sur le mal et son expression dans le conte. Don Pedro voit dans le conte l'art de trouver une conclusion. Il revient d'une guerre qu'il vient de conclure. Il parle de cette «ended action» (I.1.277) et il rêve d'appliquer le schéma de la guerre au temps de la paix. Pour ce faire il se transforme en «love-god» (II.1.364) et cherche à être l'artisan d'une fin glorieuse à l'histoire d'amour entre Claudio et Hero. Pour qu'elle puisse trouver une heureuse conclusion, cette histoire d'amour, «amorous tale» (I.1.305) sera un conte.

Was't not to this end

That thou began'st to twist so fine a story ? (I.1.289-290)

dit Don Pedro à Claudio - et quelques lignes plus loin, il conclut : «the conclusion is, she shall be thine» (I.1.307). Don Pedro joue le rôle périlleux de conteur de la vie des autres et échoue, comme échouera cet autre conteur de la vie amoureuse, Iago, qui trame les sinistres récits de sa jalousie :

For I will make him tell the tale anew

Where, how, how oft, how long ago and when

He has, and is again to cope your wife.

(Othello, IV.1.84-86)

Much Ado about Nothing est déjà un conte d'hiver. Lady Mary réussit à 
circonscrire le mal en l'enfermant dans la structure finie du conte. Shakespeare sait que le mal ne peut être si facilement circonscrit, qu'il ne peut y avoir de conclusion au mal. Si l'on cherche par orgueil à lui en imposer une, ce ne peut être qu'une pirouette de style digne des constructions finies et policées de l'euphuisme. Imposer une fin au conte de la vie ne peut être que l'œuvre de la Providence.

Mais rien n'est plus insupportable pour les scélérats shakespeariens que de laisser à la Providence le soin de leur révéler à son gré la fin de leur histoire. C'est pourquoi ils se transforment volontiers en conteurs pour présumer de cette fin dont ils veulent l'absolue maîtrise, marquant ainsi leur dépendance envers le premier des péchés : l'orgueil. Le temps de la paix dans le monde renversé des méchants, leur est insupportable tant il symbolise pour eux cette inertie devant le destin qui les pousse à l'acte fébrile, voire criminel. C'est la paix, «this weak piping time of peace» (Richard III, I.1.24) qui souffle au duc de Gloucester, le futur Richard III, les intrigues, les «plots» (I.1.32) qu'il rêve de voir aboutir en des conclusions sanglantes. N'est-il pas en effet plus facile, comme le dit le poète Wallace Stevens dans Notes Towards a Supreme Fiction de mettre un terme à la guerre militaire qu'à cette autre guerre «that never ends», «between the mind / And sky, between thought and day and night» (voir appendice).

Un sentiment d'incomplétude pousse les scélérats shakespeariens au mal, comme si le mal seul pouvait mettre un terme à cette insatisfaction originelle. Richard III ne finira jamais de souffrir de sa naissance prématurée dans un monde qui ne l'attendait pas :

Deformed, unfinished, sent before my time

Into this breathing world, scarce half made-up [...]

(Richard III, I.1.20-21)

Et Macbeth ne finira jamais d'être hanté par ce temps présent qu'il n'assume pas et qu'il rêve d'engloutir dans le souvenir nostalgique d'un passé où il pensait que le crime mettait un terme définitif à l'insatisfaction primordiale.

The time has been,

That when the brains were out, the man would die,

And there an end.

(Macbeth, III.4.77-79)

On peut interpréter de la même façon la tentation suicidaire de Hamlet, qui ose souhaiter la fin de ses souffrances sur terre.

Take arms against a sea of troubles, 
And by opposing end them ? To die to sleep -

No more ; and by a sleep to say we end

The heartache and the thousand natural shocks

That flesh is heir to.

(Hamlet, III.1.60-63)

Comme un lecteur sous l'emprise de l'urgence de connaître la fin du récit, que Kermode compare à certains malades mentaux «who have no ability to wait for the gratification of a desire» ${ }^{18}$, pour Shakespeare, l'homme sous l'emprise du mal souffre de l'impatience de mettre un terme au mal sans fin que représente par exemple Don John dans Much Ado, dont la volonté a été pervertie par une mélancolie illimitée qui lui fait refuser le remède de la raison. Conrad dit de Don John qu'il est «out of measure sad» (I.3.52). La morale de l'histoire est que la seule conclusion à laquelle on puisse arriver est celle de Benedick, enfin assagi : «Man is a giddy thing» - conclusion qui n'en est justement pas une, puisqu'elle s'ouvre sur un vertige de possibilités - mais, en tout cas, conclusion qui est à l'extrême inverse de la rigidité du scélérat qui ne s'imaginait pas pouvoir changer, comme le suggère son mépris pour la mode ${ }^{19}$. Conclusion empreinte d'une sagesse que Montaigne aurait reconnue comme la sienne, lui qui constatait combien «les moindres choses du monde tournevirent (notre jugement) ${ }^{20} \gg$.

Shakespeare illustre les grands principes de la morale thomiste sur la volonté, mais sa position se double d'un pessimisme inspiré de Montaigne quant à la part qu'il laisse à la raison. (On peut se demander si cette défiance par rapport à la raison séparée de la foi, ne va pas dans le sens d'un retour à l'augustinisme que la Réforme encourageait). Quoi qu'il en soit, Shakespeare s'inspire des bases de la doctrine chrétienne. Bien que notre nature ait été créée parfaite (Romeo and Juliet, II.3.11-12) ${ }^{21}$, Shakespeare nous met toujours en garde contre un désir de perfection excessif et nous rappelle notre nature bigarrée : «the web of our life is of a mingled yarn, good and ill together ; our virtues would be proud if our faults whipped them not, and our crimes would despair if they were not cherished by our virtues» (All's Well that Ends Well, IV.3.67-71).

Shakespeare se souvient comme Montaigne qu'à la source du mal moral, il y a la faiblesse du jugement, de la raison. C'est l'origine du scepticisme de Montaigne qui donne une cause corporelle à cette faiblesse de notre plus haute faculté : «Il est certain que nostre appréhension, nostre jugement et les facultés de nostre âme en général souffrent selon les mouvements et altérations du corps, lesquelles altérations sont continues» ${ }^{22}$.

\section{Le conte sans fin du corps}


Notre corps est de connivence avec les mouvements sans fin de la mer cruelle (Othello, V.2.363) qui se dessine toujours sur la toile de fond des tragédies shakespeariennes. Le sentiment de l'infini du mal nous vient de la perception de notre corps. Dans Hamlet, cette image est reprise comme explication au mal :

The o'ergrowth of some complexion

Oft breaking down the pales and forts of reason.

(Hamlet Q2, I.4.11-12)

«Complexion» signifie les humeurs qui constituent le corps, c'est la «complexion molle et poisante» de Montaigne ${ }^{23}$. Dans King Lear, la même explication est donnée à l'origine du mal: la promiscuité avec le corps déstabilise la raison, la fait sombrer dans le délire infini de la folie

We are not ourselves

When Nature, being oppress'd, commands the mind To suffer with the body.

(King Lear, II.4.107-109)

Le corps est ce lieu suspect dont les maladies empiètent sur l'âme. «Evil» signifiait à la fois le mal et la maladie, et le mot «illness» qui, dans Macbeth voulait dire le mal (I.5.19) finira à la fin du XVIIe siècle par signifier aussi maladie (O.E.D.). Selon la formule de Bernard Sichère, le corps est «ce réel opaque qui vient borner l'essor magnifique de la raison ${ }^{24} »$. Le corps, du côté de tout ce qui s'oppose à la raison, est le désir sombre, les humeurs troubles, tout ce qui nous attire vers la bestialité, et qui s'exprime par le langage de l'imagination. Le conte, avec ses flux et ses reflux, ses métamorphoses de vie et de mort, sa capacité à se répéter est le discours du corps en proie à toutes les contorsions de l'imagination. Le temps du conte est le temps même de la vie corporelle qui, livrée à elle-même, n'est qu'un graduel pourrissement :

And then from hour to hour we rot and rot And thereby hangs a tale.

(As You Like It, II.7.28)

Le conte est toujours le conte du corps, de sa mortalité, de la lassante logique de son dépérissement. Dans Coriolanus, le ventre, lieu de toutes les concupiscences devient le narrateur d'un conte, «a pretty tale» (I.1-88) qui narre le parcours de la nourriture dans l'espace cosmique du corps : 
I send it through the rivers of your blood

Even to the court the heart, to the seat of the brain

And through the cranks and offices of man

The strongest nerves and small inferior veins

From me receive that natural competency

Whereby they live.

(Coriolanus, I.1.132-138)

Et Falstaff, réduit bientôt à n'être que la somme de ses appétits, est connu pour sa capacité à inventer les plus étranges récits (1 Henry IV, V.4.153). Dans un état d'extrême maladie et de faiblesse, le corps de l'homme devient le parchemin sur lequel s'est inscrit le conte de sa vie.

\section{I am a scribbled form, drawn with a pen \\ Upon a parchment and against this fire \\ Do I shrink up.}

(King John, V.7.33-36)

Peter Greenaway dont l'inspiration est souvent shakespearienne, construit son dernier film The Pillow Book autour de l'image frappante d'un corps devenu parchemin et que le feu peut détruire si l'homme a l'audace de prendre la place de Dieu en signant l'œuvre de sa création - en transformant sa vie en conte.

Macbeth décrit le terrible destin de celui qui transforme sa vie en conte. La croyance en la Providence n'est pas assez forte pour conjurer l'infinie incertitude de son destin. Il veut connaître la fin de l'histoire. Il faut que sa vie devienne un conte avec un début, un milieu et une fin, et la fin est pour lui l'accès au pouvoir. Macbeth auparavant n'aimait-il pas les contes ?

The time has been, $[\ldots]$

[...] my fell of hair

Would at a dismal treatise rouse and stir,

As life were in't.

(Macbeth, V.5.10-13)

Alors, il se laisse tout entier envahir par une imagination nourrie de ses «black and deep desires» (I.5.51). Son imagination donne une réalité aux prophéties vides des sorcières, «as if life were in them». Mais cette matérialité fictive, cet excès de sens qu'il imprime à ces bulles vides que sont les sorcières, va petit à petit vider sa vie de substance, la rendre non pas achevée comme il le pensait, mais interminable comme l'incertitude qu'il voulait 
conjurer. Par un curieux retour, le conte achevé qu'il voulait faire de sa vie devient un conte sans fin, un conte dont la fin est éternellement différée. (Kermode a montré que la procrastination de la conversion de saint Augustin a peut-être inspiré le triple «tomorrow» (cras) de l'acte V) ${ }^{25}$. Même la mort de la reine n'est plus qu'un mot pour lequel il n'y a plus de place dans le déroulement du temps. La mort désormais habite le langage, et les mots du langage sont vides comme elle, «signifying nothing», pour avoir trop donné de sens aux prophéties.

Macbeth atteint ce «collapse of language», dont parle Oppenheimer, qui décrit le mal comme «a complete speechlessness, a death silence» ${ }^{26}$. Il est devenu un être de fiction, «a monster» «painted upon a pole» (V.8.26) tandis que Lady Macbeth, transformée en conteuse répète inlassablement les paroles fatales de sa vie qui l'ont amenée à cautionner «a deed without a name» (IV.1.49). Le conte n'est-il pas, comme Freud l'avait remarqué, l'art de la répétition ${ }^{27}$ et Shakespeare faisait dire au Dauphin Louis que «life is as tedious as a twice-told tale» (King John, III.4.108).

En faisant dire le mal au conte, Shakespeare s'inscrit dans une logique que nous reconnaissons bien. Le conte a toujours été suspect, des scolastiques à Descartes. Il n'est pas surprenant dès lors que le scélérat soit presque toujours représenté comme un conteur qui détruit la subtile hiérarchie de ses facultés en laissant les excès des humeurs et de l'imagination dominer la raison.

C'est pourquoi Myriam Revault d'Allonnes est justifiée de dire que le drame shakespearien donne à voir la pensée traditionnelle sur le problème du mal «sa dimension démoniaque ou diabolique, toute méchanceté essentielle, toute malfaisance innée, et plus généralement tout mobile ancré dans la dépravation, la convoitise et autres passions obscures» ${ }^{28}$.

Pourtant, après avoir lu ses chapitres sur la banalité du mal au XXe siècle, sur ce qui fait la spécificité du mal moderne par rapport au mal que décrit la pensée traditionnelle, cette capacité à créer un enfer qui se définirai comme «ce lieu où la vie disparaît alors que l'homme est encore vivant» (p. 16) ${ }^{29}$, il m'a paru intéressant de rechercher dans l'œuvre de Shakespeare des indices de ce qu'allait être le mal pour l'homme du XXe siècle.

Le «lac de noirceur»: un mal sans rime ni raison?

Dans les pièces de Shakespeare existe-t-il ce que l'on pourrait appeler des espaces limites où l'on serait confronté non pas à une simple faillite de la raison, mais à une absence de raison et à un sursaut particulièrement agressif de la volonté qui serait pure volonté du mal. Il semble alors que se déchirerai la correspondance vitale que saint Thomas établissait entre le jugement et la volonté humaine. Dans sa description du mal, Shakespeare va-t-il plus loin que le scepticisme de Montaigne qui finalement ne permettait que de mieux 
resouder la volonté à ce qui fonde la hiérarchie des facultés supérieures de l'homme, ce qui unit en définitive la volonté, la raison et l'âme ${ }^{30}$.

Tentons donc de parcourir ces espaces de l'œuvre à la recherche d'exemples où il n'y aurait pas de raison au mal, où la volonté serait dotée d'un pouvoir infini, capable de détrôner la raison de son rôle de modérateur et de mobile ultime de l'acte. Y-a-t-il donc un espace où Shakespeare se serait risqué à décrire la limite extrême d'abord tracée par les nominalistes qui, dans leur théorie de la volonté, tentaient de séparer ce que saint Thomas avait uni. Y-a-t-il un espace, où la volonté pouvant devenir volonté du mal, rejoindrait la définition du «fortuit» donnée par Gilson ${ }^{31}$ ou, en d'autres termes, y-a-t-il dans l'œuvre un lieu où s'opère la perte du référent téléologique dont parle Myriam Revault d'Allonnes ${ }^{32}$ ?

Lorsque Shakespeare décrit le mal, va-t-il chercher plus loin que le sceptique Montaigne les raisons de la faiblesse de la raison ? Se limite-il à l'attribuer à sa proximité avec les fluctuations infinies du corps ? Ne met-il pas aussi en scène l'expression d'un désir affranchi du contrôle de la Raison et qui n'aurait pas de conséquences néfastes pour l'homme mauvais? Il n'y a pas de scélérat gagnant chez Shakespeare, mais pourtant commence à poindre l'idée d'un désir assimilable non plus à la concupiscence, mais à une force vitale dont les effets ne seraient pas en conflit avec la Nature. Dans King Lear, Edmund décrit le désir comme une force vitale qui va presque jusqu'à donner au Mal des lettres de noblesse.

Why bastard? Wherefore base?

When my dimensions are as well compact,

My mind as generous, and my shape as true

As honest Madam's issue ? Why brand they us

With base ? with baseness ? bastardy? base, base ?

Who in the lusty stealth of nature take

More composition and fierce quality

Than doth, within a dull, stale, tired bed,

Go to the creating a whole tribe of fops,

Got 'tween asleep and wake.

(King Lear, I.2.6-15)

Certes, la nature dont parle Edmund ici n'est pas la Natura naturans, mais la Natura naturata qu'oppose W.R. Elton dans King Lear and the gods, et il faut en tenir compte pour ne pas tirer des conséquences fausses. Quoi qu'il en soit, nous sommes loin de la description du mal chez Richard III où le désir mauvais imprime sur le corps les marques de sa difformité. On voit comment la pensée en ce début du XVIIe siècle s'achemine peu à peu vers la transformation radicale du discours éthique que l'on trouvera quelque cinquante 
ans plus tard chez Hobbes, lorsqu'il refusera la définition scolastique de la volonté, la séparera définitivement de la Raison pour l'isoler du côté du désir, fera d'elle l'aboutissement d'une délibération motivée par un désir devenu force vitale, débarrassé de connotations diabolisantes ${ }^{33}$.

Shakespeare ne libère pas le désir de son commerce avec le mal comme Hobbes et la pensée moderne chercheront à le faire. Le désir reste dans son œuvre la force souterraine et sombre, assimilable au pouvoir infini du mal Macbeth est habité par ces «black and deep desires» (I.4.51) qui relient l'homme à des profondeurs insondables, à ce «lac de noirceur» (King Lear, III.6.7) que l'on pressent sans fond ${ }^{34}$. Sans fond, mais neutralisable par la capacité de l'homme à se laisser envahir par la grâce tout aussi infinie de Dieu, sans fond mais explicable, sans fond mais gouvernable par l'assaut conjoint de la volonté et de la raison.

Pourtant, cette pensée traditionnelle sur le mal est comme poussée dans ses derniers retranchements dans l'œuvre de Shakespeare, au point que l'on peut se demander si Shakespeare ne se ferait pas l'écho d'une pensée déjà moderne dans sa désespérance, jamais totale toutefois, de la maîtrise du mal. J'ai relevé trois exemples qui me paraissent signifiants des cas où la raison est menacée : dans sa difficulté à nommer la cause du mal, dans sa difficulté à maintenir au-delà du mal extrême une finalité, un référent téléologique - dans une éventuelle incapacité du langage à dire le mal. André Green a pu dire qu'à l'origine des trois grandes figures du mal chez Shakespeare, Richard III, Iago et Edmund, il y avait toujours une guerre fratricide qui expliquait le mal. Par contre, il écrit : « la soif de meurtre de Macbeth est sans explication ${ }^{35}$ ». S'il n'y a pas en effet d'éclaircissement explicite dans le texte, il est facile toutefois de trouver à ces pulsions des origines dans l'orgueil qui pousse Macbeth à vouloir connaître son destin, à vouloir être le conteur de sa vie et donc à faire rentrer le scélérat dans les schémas traditionnels dont parle $\mathrm{M}$ Revault d'Allonnes. Green veut dire que Shakespeare ne prend pas la peine, dans ce cas, de trouver une explication psychologique au mal.

C'est que justement Shakespeare est fasciné par l'absence de cause psychologique. Sans cesse, nous trouvons ce thème qui mit si souvent à ma la critique psychologisante traditionnelle et fait entrer Shakespeare dans la problématique moderne du mal. Dans sa conférence annuelle sur Shakespeare à la British Academy de 1995, Alastair Fowler mettait l'accent sur cette pierre d'achoppement de la critique ${ }^{36}$. Le mot «cause» ponctue toutes les grandes tragédies shakespeariennes qui sont toutes des tentatives pour trouver des causes au mal et se moquer de celles qui viennent trop facilement à l'esprit. L'obtus Polonius est un champion de cet exercice lorsqu'il s'obstine à voir dans l'amour de Hamlet pour sa fille la cause de sa folie. Claudius pour une fois nous inspire du respect, qui ne trouve rien d'autre que le terme «something» (Hamlet, III.1.165), si proche de «nothing» pour dire 
l'indéfinissable folie. De cette quête en amont du mal, il reste l'image de Hamlet qui pense aux armées détruites sans raison et déplore qu'il y ait «no cause without why the man dies» (IV.4.29-30). P. Di Mascio commentait ce vers en concluant : «le mal existe parce que ce rien existe» ${ }^{37}$.

Les causes du mal dans Shakespeare sont indéfinissables, innommables lorsque les scélérats sont tentés d'y avoir recours comme justification à leur crime. L'innocence de Desdémone rend la cause du meurtre d'Othello inexistante.

It is the cause, it is the cause my soul

Let me not name it to you you chaste stars.

(Othello, V.2.1-2)

Emilia soulignait déjà l'absence de cause dans son dialogue avec Desdémone :

Des. Alas the day, I never gave him cause!

Emil. But jealous souls will not be answer'd so ;

They are not ever jealous for the cause,

But jealous for they are jealous : 'tis a monster,

Begot upon itself, born on itself.

(Othello, III.4.156-160)

Tautologie monstrueuse du mal, absence de raison, aberration. La volonté du mal ici n'acquiert-elle pas une autonomie troublante? On peut objecter que la théologie médiévale avait même trouvé une place pour les monstres dans le plan divin et que qualifier le mal de monstrueux permettait déjà de l'intégrer dans une conception du monde à visée téléologique. Mais comme me le fit remarquer Gisèle Venet, l'image du monstre est volatilisée lorsqu'Othello, cherchant sur le corps de Iago des traces de sa monstruosité diabolique (en l'occurence les pieds fourchus), ne les trouva pas (V.2.287288). Preuve s'il en faut, d'une fascination toute moderne pour un mal sans explication.

Shakespeare ne cherche-t-il pas à isoler le mal de toute référence structurante, rassurante, à lui donner une autonomie par rapport au pouvoir du bien ? Mais, on constate que les grandes victoires scandaleuses du mal, la mort de Cordélia par exemple, sont dues à l'erreur, ressort classique de la tragédie. Ainsi, en cherchant les causes du mal, Shakespeare l'attribue parfois à l'erreur comme pour signaler que le mal absolu se conçoit toujours dans un rapport - même négatif - avec la raison puisqu'une erreur ne peut être définie que dans un contexte rationnel.

Nous avons déjà parlé avec Montaigne d'un scepticisme appliqué à 
fragiliser la raison. J'y reviens avec un exemple qui met en cause le référent téléologique lui-même. C'est la thèse de l'Élégie Funèbre composée en l'honneur de William Peter, assassiné le 25 janvier $1612^{38}$ (voir appendice). Je n'entrerai pas ici dans la polémique sur la paternité de l'œuvre, mais j'utiliserai ce «tale of woe» (v. 167), parce que même s'il s'avère qu'il n'est pas de Shakespeare, il pose en termes proches de notre analyse, un aspect particulier de la problématique du mal. L'assassinat du jeune homme, «his predestinated end» (v. 1) a détruit ce qui donnait sens à sa vie, l'espoir qu'il avait mis dans son avenir, la finalité qu'il s'était donnée et le génie du poète consiste à faire rimer «end» (v. 1) avec «intend» (v. 3) : «both his youth and virtue did intend / The good endeavours of deserving praise». Cette même rime ne réapparaît qu'une fois dans le poème, beaucoup plus loin. Le mot «end» (v. 360) se réfère toujours à ce meurtre terrible, mais cette fois-ci le mot «intend», lui servant de rime (v. 362), décrit les mauvaises intentions de ces calomniateurs qui tirent parti de l'assassinat pour atteindre la réputation du jeune homme. Ce parallélisme formel souligne l'opposition entre les bonnes intentions de William Peter et les mauvaises intentions de ses calomniateurs, encore plus criminels que les assassins, puisqu'ils s'attaquent aux raisons de vivre du jeune homme, à la finalité de sa vie. La même situation se retrouve dans Coriolanus où l'un des conspirateurs suggère à Aufidius d'annihiler définitivement Coriolan en racontant sur lui des calomnies qui enlèveront à sa vie tout son sens. Il s'agit d'enterrer avec son corps les «raisons» de sa vie, c'est-à-dire la finalité qu'il s'était donnée.

When he lies along After your way his tale pronounced shall bury His reasons with his body.

(Coriolanus, V.6.57-59)

Une vie qui ne peut répondre d'un sens, d'un rapport au Logos fondateur n'a plus de raison d'être. Enlever à un mort cette cohérence, «les raisons» de sa vie, c'est le crime parfait. Les tombes souvent anonymes de notre siècle, disent comme ces deux textes que tel est bien le mal absolu, le meurtre de ce qui relie l'homme à une finalité.

Le poète dénonce le raisonnement captieux des calomniateurs coupables d'un rationalisme pervers où n'entrerait aucune religion et déplore les temps où «men were only led by Reason's law» (v. 365). C'est en effet cette dépendance envers le raisonnement du proverbe : «such is the end the life proves so» (v. 366) qui amène les ennemis du jeune homme à justifier leur calomnie. Ils tirent prétexte de ce meurtre pour s'instaurer en conteurs de la vie de William Peter, pour récrire sa vie à rebours à la manière d'un conte don on sait la fin. Nous reconnaissons là la fascination pour la fin, dont nous 
avons parlé et qui caractérise les scélérats shakespeariens ainsi que le bouleversement du temps qui en découle. Déterminer la vie par rapport à la fin, c'est dénier le pouvoir de la Providence, c'est s'instaurer en dieu tout puissant, maître du destin de l'autre, c'est refuser l'incertitude positive qui fonde la foi.

Ainsi le poète oppose la rime à une raison qui se voudrait toute puissante : la répétition de cette double rime structure la cohérence du poème en en faisant peut-être le dernier mot de la réponse shakespearienne au mal.

Le troisième exemple décrit la possibilité de la mort du Logos lui-même, la limite ultime. Dans sa prison de Pomfret, Richard II reprend à son compte tous les arguments dont Dame Philosophie avait fait profiter Boèce. Alliant les facultés supérieures, le cerveau, décrit comme le principe femelle, à l'âme, le principe mâle (V.5.6-7), Richard engendre des pensées qui avaient permis à Boèce de tenir tête au mal infini de la torture et de la mort. Mais c'est à se demander si Bolingbroke n'est pas un adversaire encore plus implacable que le roi des Goths, puisque sa volonté semble venir à bout de la Raison même. Dans la prison de Pomfret, le florilège de la Raison s'étiole, les mots ne s'arriment plus dans la réalité. Ils rassemblent les pensées dans les intrigues improbables de contes sans fin : «Thoughts... do plot / Unlikely wonders» (V.5.18-19). C'est la faillite du langage, de la pensée devant la menace du nouveau monde d'un nominalisme caricatural, où les mots échappent au contrôle de la volonté, passent à travers les remparts des geôles et tuent, inspirent des soliloques impuissants pour se moquer du pouvoir même de la poésie en en faisant une dérisoire alternative au pouvoir politique. Dans le vertige de cette dissolution, le corps du roi n'est plus qu'un cadavre, ce que Tertullien décrivait comme cette chose qui ne porte aucun nom dans aucune langue, la seule chose qui n'ait pas de nom, qui résiste au langage. C'est la «mort muette» (I.3.170-174).

Debora Shuger fait le constat d'un recul de l'influence de la patristique à la Renaissance. Shakespeare semble s'en faire l'écho. Il n'y a plus de place dans le langage même pour la compassion, puisque dans cette nouvelle société sécularisée, les pauvres ne sont plus décrits comme les membres du corps souffrant du Christ, mais comme une gêne, voire une menace contre le pouvoir ${ }^{39}$. La souffrance de l'individu n'a pas de répondant. Richard disparaît dans le silence insupportable du langage qui se meurt, de mots qui ne peuvent plus exprimer la souffrance, ni appeler la compassion de quiconque.

Les tragédies de Shakespeare vont jusqu'à décrire comment le langage même peut mourir, il décrit le processus qui peut aboutir à ébranler ce que Shakespeare considérait comme le dernier rempart contre le mal. Edgar disait : «The worst is not so long as we can say this is the worst» (King Lear, IV.1.27-28), rappelant ainsi la victoire du Logos sur la fascination des contes sans fin du mal. A Pomfret, faute de mots, la musique se fait entendre, dernier 
refuge de l'harmonie du monde.

Dans son dernier chapitre de The Sense of an Ending, Frank Kermode s'est servi de la scène de Pomfret pour illustrer le combat victorieux de Christopher Burney, cet agent britannique emprisonné par les nazis, qui dans un isolement total, jouait comme Richard les mots contre les mots, mais à la différence du roi médiéval affirmait ainsi la victoire du Logos sur le mal absolu, la volonté d'un total asservissement.

«Our revels are now ended», et Prospero laisse le dernier mot à la Providence. Mais pas d'optimisme béat pourtant: le texte shakespearien sonde le «lac de noirceur» et conclut que le mal est d'abord l'Orgueil L'orgueil transforme les hommes insatisfaits de leur sort en conteurs satisfaits, qui mettent un terme ainsi à l'incomplétude insupportable de leur vie, sans se rendre compte qu'ils ne font qu'écrire les contes sans fin du mal. Devant ce constat théologique, cette approche morale du mal, nous pourrions nous demander si Shakespeare n'avait pas les mots pour dire le mal sans cause de notre contemporanéité.

Pourtant la galerie terrifiante des «unfinished faces» de ses scélérats j'emprunte cette formule à Philip Larkin qui s'en sert ici pour décrire le visage de la folie contemporaine ${ }^{40}-$ s'ouvre sur l'univers fade, kafkaïen, répétitif que nous reconnaissons bientôt comme le nôtre, où le sujet, n'existant pas encore, et ne trouvant pas dans un ailleurs le reflet de son identité, s'affirme dans le mal.

Incapable de mesurer «the thousand natural shocks / That flesh is heir to» (Hamlet, III.1.63-64), n'osant pas prendre la mesure de l'infini du mal, niant parfois même, ne rendant pas au diable ce qui revient au diable, les scélérats shakespeariens ressemblent parfois déjà plus à nos utopistes totalitaires qu'aux Vices du moyen âge.

C'est que dans l'inachèvement de ces contes du mal, nous voyons poindre une conscience déjà moderne d'un mal sans rime ni raison, même si Shakespeare cherche à reconstruire encore et toujours, les «pales and forts of reason» (Hamlet, I.4.11-12), qui sans cesse s'écroulent, avec les moyens de la morale traditionnelle. 


\section{${ }^{\mathrm{N}}$ O T E S}

${ }^{1}$ Coriolanus, I.3.60-66 et IV.6.95.

So we'll live and pray and sing and

Tell old tales and laugh at guilded butterflies

(King Lear, V 3.11-13)

${ }^{2}$ P. Ricœur, Temps et Récit, Seuil, 1983. Vol.2, p. 49. F. Kermode, The Sense of an Ending, O.U.P., 1967, p. 6.

${ }^{3}$ N. Frye, Anatomy of Criticism, Princeton University Press (1957), 1973, p. 117.P. Ricœur, p. 39.

${ }^{4}$ F. Kermode, The Sense of an Ending, O.U.P., 1967, p. 13

${ }^{5}$ G.R. Hibbard, ed., Hamlet, The World's Classics, O.U.P., 1994, p. 353, note 318.

${ }^{6} \mathrm{~B}$. Sichère m'a fait part des références dans le Traité des Principes (II.10.3 ; III.5.7 ; III.6.7) où, à la différence de sa position dans Contre Celse, Origène avait imaginé que même le mal extrême en la personne de Satan serait, lors du Jugement Dernier, racheté dans la mesure où il serait contradictoire de penser que quelque réalité que ce soit pût encore résister à l'amour infini de Dieu. Voir B. Sichère, Histoires du Mal, Grasset, 1996, p. 117. Voir aussi Tobias Smollett, The Expedition of Humphry Clinker, 1771, Lettre de Jeremy Melford, 3 septembre. (allusion à ce débat qui faisait rage à la fin du XVIIIe siècle).

${ }^{7}$ G. Minois, L'Histoire des Enfers, Fayard, 1991, p. 106-107.

${ }^{8}$ G. Duby, La Fin des Temps, Stock, 1982, p. 175.

${ }^{9}$ G. Duby, p. 9 .

${ }^{10} \mathrm{~K}$. Thomas, Religion and the Decline of Magic, Penguin, 1971 ,p. 471 et 462.

${ }_{11}$ G. Duby, p. 30.

${ }^{12}$ Y. Peyré, «Les Masques d'Ariel, Essai d'interprétation de leur symbolisme», Cahiers Elisabéthains, 19, 1981, p. 53-71.

${ }^{13}$ V. Harris, All Coherence Gone, The University of Chicago Press, 1966 (1949). «This great world / Shall so wear out to naught» (King Lear, IV .6.136-137).

${ }^{14}$ W.C. Curry, Shakespeare's philosophical Patterns, Louisiana State University, 1959 (1937), p. 231. 
${ }^{15}$ B. Castiglione, The Book of the Courtier, G. Bull ed., Penguin Classics, 1980 (1967), p. 292.

${ }^{16}$ H. Weinrich, Le Temps, Seuil, Paris, 1973 (1964), p. 164.

${ }_{18}^{17}$ H. Weinrich, p. 66.

${ }^{18}$ F. Kermode, p. 55.

${ }^{19}$ Voir l'apologie de la mode dans The Book of the Courtier, G. Bull ed., p. 110-111.

${ }^{20}$ Apologie de Raimond Sebond, Montaigne, Euvres Complètes, Rat. M. ed., La Pléiade, Gallimard, Paris, 1962, p. 547.

${ }^{21}$ R. Mushat Frye, Shakespeare and Christian Doctrine,

Princeton University Press, 1963, p. 217.

${ }_{22}^{2}$ Montaigne, p. 547.

${ }^{23}$ Montaigne, p. 552

${ }^{24} \mathrm{~B}$. Sichère, Eloge du Sujet. Du retard de la pensée sur les corps, Grasset, Paris, 1990, p. 48.

${ }^{25}$ P. Ricœur, p. 48.

${ }^{26}$ P. Oppenheimer, Evil and the Demonic, a new theory of monstrous behaviour, Duckworth, London, 1996, p. 18.

${ }^{27}$ S. Freud, Beyond The Pleasure Principle, J. Strachey ed., The Hogarth Press, London, 1974, p. 29 : «If a child has been told a nice story, he will insist on hearing it over and over again rather than a new one, and he will remorselessly stipulate that the repetition shall be an identical one and will correct any alterations of which the narrator may be guilty».

${ }^{28} \mathrm{M}$. Revault d'Allonnes, Ce que l'homme fait à l'homme, Seuil, 1995, p. 22.

${ }^{29}$ M. Revault d'Allonnes, p. 16.

${ }^{30}$ Montaigne, la conclusion de l'Apologie de Raimond Sebond, p. 589.

${ }^{31}$ E. Gilson, L'Esprit de la Philosophie Médiévale, Vrin, Paris, 1978, p. 348-349.

${ }_{32}$ M. Revault d'Allonnes, p. 109-110.

${ }^{33}$ Ces remarques sur Hobbes m’ont été suggérées par l'analyse de Frank Lessay au cours de sa communication «Hobbes et les Passions» dans le cadre du séminaire d'Epistemè, dirigé par Gisèle Venet (21 octobre 1996). Voir Leviathan, 1ère partie, chapitre 6. Voir aussi F. Lessay ed., Hobbes, De la liberté 
et de la nécessité, tome XI-1 des Oeuvres de Hobbes, Vrin, Paris, 1993.

${ }^{34}$ Edith Sitwell dans A Notebook on William Shakespeare compare cette citation à celle de Measure for Measure (III.1.9697) où, parlant d'Angelo, Isabella dit : «This filth within being cast, he would appear / A pond as deep as hell». Voir K. Muir ed., King Lear, The Arden Shakespeare, Methuen, 1968, p. 130.

A. Green, «Pourquoi le Mal ?», Nouvelle Revue de Psychanalyse, $\mathrm{n}^{\circ} 38$, Gallimard, automne 1988, p. 252.

${ }^{36}$ A. Fowler, «The Case against Hamlet», $T L S$, Déc. 22nd 1995 , p. 6-8. Voir M. Jones-Davies, «Ophélie : identification d'une femme», H. Suhamy ed., Hamlet, Ellipses, 1996, p. 116125. Aussi : M. Jones-Davies «La cruauté de Hamlet et le silence d'Harpocrate», in P. Di Mascio ed., L'auteur à l'œuvre, ENS, Éditions Fontenay/Saint Cloud, 1996, p. 59-85.

${ }^{37}$ P.Di Mascio, «Hamlet et le texte freudien: phénoménologie de l'interprétation», in P. Arnaud ed., Regards sur la critique littéraire moderne, Presses de l'Université de Paris-Sorbonne, Paris, 1996, p. 59.

${ }^{38}$ Donald, W. Foster ed., W[illiam] S[hakespeare], Élégie Funèbre, trad. Lucien Carrive, Stock, Paris, 1996.

${ }^{39}$ Debora K. Shuger, «Subversive fathers and suffering subjects : Shakespeare and Christianity», inD. B. Hamilton, \& R. Strier ed., Religion, Literature and Politics in Post-Reformation England, 1540-1688, Cambridge University Press, 1996, p. 55.

${ }^{40}$ Philip Larkin, «Neurotics», 1949. 


\section{A P P E N D I C E}

Soldier, there is a war between the mind

And sky, between thought and day and night. It is For that the poet is always in the sun, 
Patches the moon together in his room

To his Virgilian cadences, up down,

Up down. It is a war that never ends.

Yet it depends on yours. The two are one.

They are a plural, a right and left, a pair,

Two parallels that meet if only in

The meeting of their shadows or that meet

In a book in a barrack, a letter from Malay.

But your war ends. And after it you return

With six meats and twelve wines or else without

To walk another room... Monsieur and comrade,

The soldier is poor without the poet's lines,

His petty syllabi, the sounds that stick,

Inevitably modulating, in the blood.

And war for war, each has its gallant kind.

How simply the fictive hero becomes the real ;

How gladly with proper words the soldier dies,

If he must, or lives on the bread of faithful speech.

Wallace Stevens, «Notes Towards a Supreme Fiction» (conclusion)

\section{A FUNERAL ELEGY}

Since Time, and his predestinated end,

Abridg'd the circuit of his hopeful days,

Whiles both his Youth and Virtue did intend

The good endeavors of deserving praise,

5 What memorable monument can last

Whereon to build his never-blemish'd name 
But his own worth, wherein his life was grac'd-

$$
[\ldots]
$$

Lo, here a lesson by experience taught

350 For men whose pure simplicity hath drawn

Their trust to be betray'd by being caught

Within the snares of making truth a pawn ;

Whiles it, not doubting whereinto it enters,

Without true proof and knowledge of a friend,

355 Sincere in singleness of heart, adventers

To give fit cause, ere love begin to end :

His unfeign'd friendship where it least was sought,

Him to a fatal timeless ruin brought;

Whereby the life that purity adorn'd

360 With real merit, by this sudden end

Is in the mouth of some in manners scorn'd,

Made questionable, for they do intend,

According to the tenor of the saw

Mistook (if not observ'd, writ long ago

365 When men were only led by Reason's law),

That «such as is the end, the life proves so».

Donald W. Foster, ed., W[illiam] S[hakespeare], Élégie Funèbre, tr. Lucien Carrive, Stock, 1996. 
Margaret Jones-Davies 
